\title{
EFFECT OF CRACKING AND FRACTURE ON THE ELECTROMECHANICAL RESPONSE OF HPFRCC
}

\author{
XIAOPENG LI* AND MO LI* \\ University of California, Irvine \\ Irvine, CA, USA \\ e-mail: moli@uci.edu
}

Key words: High-performance fiber reinforced cementitious composites, Electrical impedance spectroscopy, Complex impedance, Piezoresistive effect, Crack opening, Multiple cracking, Steadystate cracking

\begin{abstract}
High-performance fiber reinforced cementitious composites (HPFRCC) feature multiple steady-state cracking before localized fracture occurs. This process depends on a variety of factors, such as the flaw distribution in the material, matrix toughness, crack bridging spring law and fiber/matrix interfacial properties. The cracking and fracture process in HPFRCC not only dictates its mechanical behavior but also strongly affects its electrical response. This paper aimed to understand these effects, which will offer fundamental tools to design new multifunctional HPFRCC intentionally encoded with strain and damage self-sensing functionalities. Two HPFRCC material systems were investigated: both exhibited strain-hardening behavior while one had modified electrical properties through the incorporation of carbon black nanoparticles. The experimental approach coupled mechanical testing with four-probe electrical impedance spectroscopy. The experimental results revealed the relation between impedance magnitude and single crack opening, which can be approximately linear or nonlinear depending on the material design. The results also showed the effects of elastic, strain-hardening and localized cracking (tension softening) stages on the impedance magnitude change as well as the complex impedance Nyquist plots, paving the way for further analysis and development of theoretical models.
\end{abstract}

\section{INTRODUCTION}

The development of HPFRCC in past decade has broadened researchers' view on cementitious materials. Unlike normal fiber reinforced cementitious materials (FRCs) with a tension-softening response, HPFRCC features a pseudo strain-hardening behavior under tension. This is achieved by integrating micromechanics theory, rheology design for processing, and microstructure tailoring. By simultaneously satisfying steady-state crack propagation criteria [1] and maximized microcracking density [2], HPFRCC shows extraordinary tensile ductility and controllable width of microcracks.
The tensile stress-strain relation in HPFRCC can be divided into three stages: 1) The initial elastic stage, characterized by Young's modulus. (2) The strain-hardening stage, accompanied by multiple steady-state microcracking formation. Once the first microcrack occurs, it propagates in a steady state. Further increase of load then activates the second steady-state microcrack in a different location. This process continues with increasingly applied deformation and load, until the applied stress reaches the maximum fiber bridging capacity. The number of microcracks that form during this stage determines the tensile strain capacity of 
HPFRCC. (3) The tension-softening stage, accompanied by the formation of a localized fracture at one of the microcracks and the continuous drop of the ambient load. At this stage, the final crack propagates as a Griffith crack rather than a steady-state crack. Undoubtedly, the cracking behavior of HPFRCC, at single crack propagation scale and at multiple cracking scale, determines HPFRCC's mechanical response under load. The effect of cracking on HPFRCC electrical response, however, has not been understood. New knowledge on the coupled mechanical and electrical properties of HPFRCC can offer fundamental tools to design new multifunctional materials [3, 4] intentionally encoded with strain and damage self-sensing functionalities.

Cementitious materials are semi-conductors. Its electrical response under AC currents depends on its pore structure and connectivity, as well as the interconnecting layers of C-S-H gels $[5,6]$. For HPFRCC, the fibers dispersed in the cementitious matrix are typically polymeric fibers that are considered as nonconductive fibers. When a crack occurs, the change in electrical impedance of HPFRCC can be captured by four-point electrical impedance spectroscopy. In this study, two HPFRCC material systems were investigated, one was a typical HPFRCC and the other was a newly developed HPFRCC incorporating carbon black nanoparticles. Experiments were conducted at single crack propagation scale, and at multiple cracking scale.

\section{EXPERIMENTAL STUDY}

\subsection{Materials}

HPFRCC is a large family of materials with various mixture designs. The mixing proportion of the HPFRCC investigated in this study is shown in Table 1. It contains Portland Type I cement, class F fly ash, fine silica sand, water and $1.8 \%$ by volume polyvinyl alcohol (PVA) fibers. The dry ingredients were first mixed with water and chemical admixtures for 1 minute to reach a homogeneous state. The fibers were then slowly added and mixed for another 2 minutes. The HPFRCC mixture was cast into coupon specimens with dimensions of $508 \mathrm{~mm} \times 50.8 \mathrm{~mm} \times 12.5 \mathrm{~mm}$ and cured for 28 days. The coupon specimens were first tested under uniaxial tension test to confirm the processed material was indeed a HPFRCC with tensile strain-hardening behavior. The test results are shown in Figure 1. The HPFRCC demonstrated a tensile strain capacity of $4.6 \%$. A large number of microcracks with close spacing formed during the strain-hardening stage, with the average crack width of $40 \mu \mathrm{m}$.

Table 1: Mix proportion of HPFRCC

\begin{tabular}{ccc}
\hline \multirow{2}{*}{ Ingredients } & \multicolumn{2}{c}{ Proportion $\left(\mathrm{kg} / \mathrm{m}^{3}\right)$} \\
\cline { 2 - 3 } & HPFRCC 1 & HPFRCC 2 \\
\hline Cement & 292 & 266 \\
\hline Fly ash & 935 & 843 \\
\hline Sand & 456 & 456 \\
\hline Water & 312 & 368 \\
\hline PVA fiber & 26 & 26 \\
\hline Superplasticizer & 2.7 & 2.7 \\
\hline Carbon black & & 25 \\
\hline
\end{tabular}

(a)

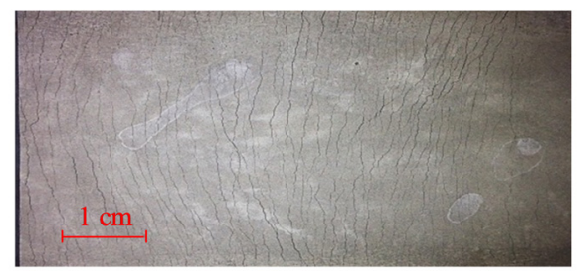

(b)

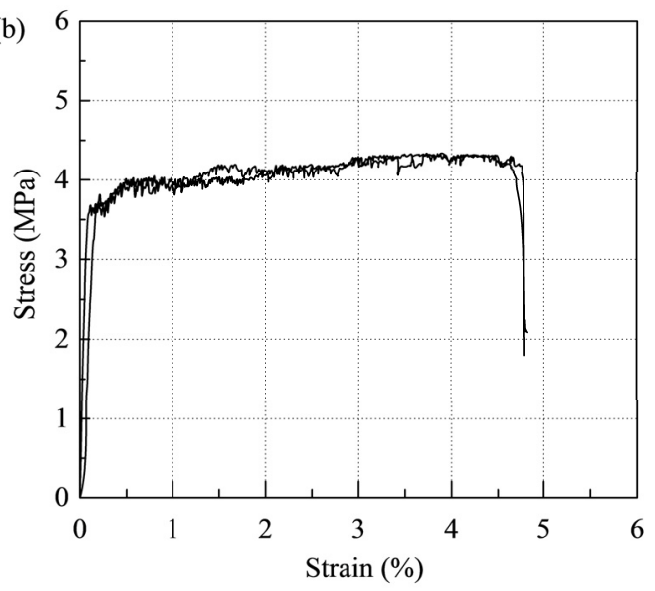

Figure 1: HPFRCC tensile behavior: (a) multiple microcracking and (b) tensile stress-strain relation.

6-inch by 3-inch cylinder specimens were 
prepared to measure the compressive strength of HPFRCC investigated in this study. The results are shown in Table 2. The average compressive strength was $44.2 \mathrm{MPa}$.

Table 2: Compressive strength of HPFRCC in this study

\begin{tabular}{cccccc}
\hline number & 1 & 2 & 3 & 4 & $\begin{array}{c}\text { Average } \\
\text { strength } \\
(\mathrm{MPa})\end{array}$ \\
\hline $\begin{array}{c}\text { Strength } \\
\text { (MPa) }\end{array}$ & 46.0 & 43.3 & 42.3 & 45.1 & 44.2 \\
\hline
\end{tabular}

\subsection{Single crack opening}

To understand the effect of single crack opening on HPFRCC electrical response, uniaxial tension test was performed on doublenotched specimens (Figure 2). The $152.4 \mathrm{~mm}$ $\times 50.8 \mathrm{~mm} \times 12.5 \mathrm{~mm}$ specimen was notched on both sides by $10 \mathrm{~mm}$ each so that under tension, a single crack formed along the notched cross section. The test was performed under uniaxial tension with displacement control at a rate of $0.003 \mathrm{~mm}$ per minute. During testing, a high-resolution digital image correlation (DIC) system was adopted for accurate measurement of crack opening (Figure 3). The specimen surface was painted with black speckles for the DIC to track the movement of the speckles during loading. The region measured by DIC was shown in blue color. The crack opening values were obtained by measuring the relative vertical displacement between two red lines through DIC, as shown in figure 3.

Four copper electrodes were attached to the surfaces of the specimen. The outer two electrodes were used to inject $\mathrm{AC}$ current, and the inner two electrodes were used for measuring voltage. The electrical response of the specimen during single crack opening was measured at a frequency of $1500 \mathrm{HZ}$ throughout the test.

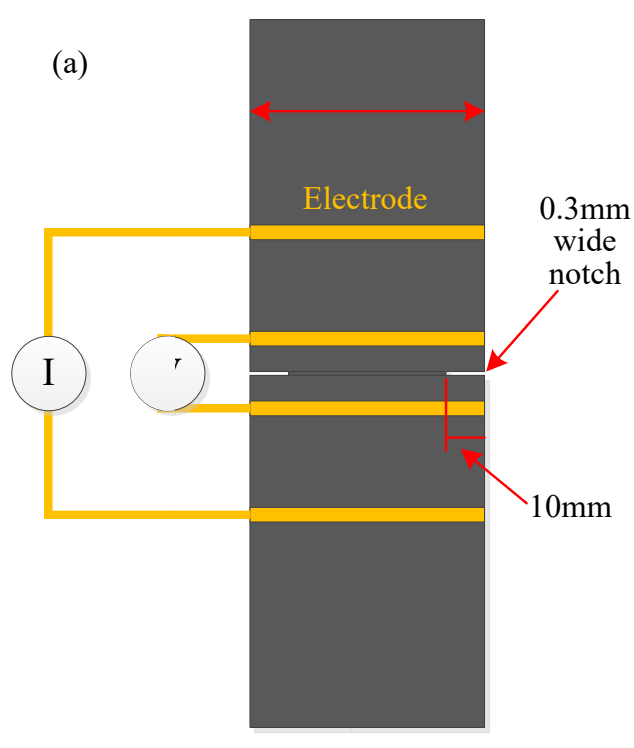

(b)

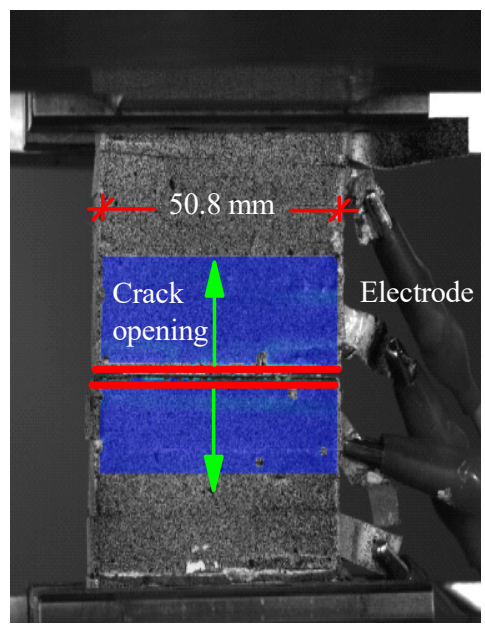

Figure 2: Single crack opening test: (a) specimen geometry, and (b) test setup.

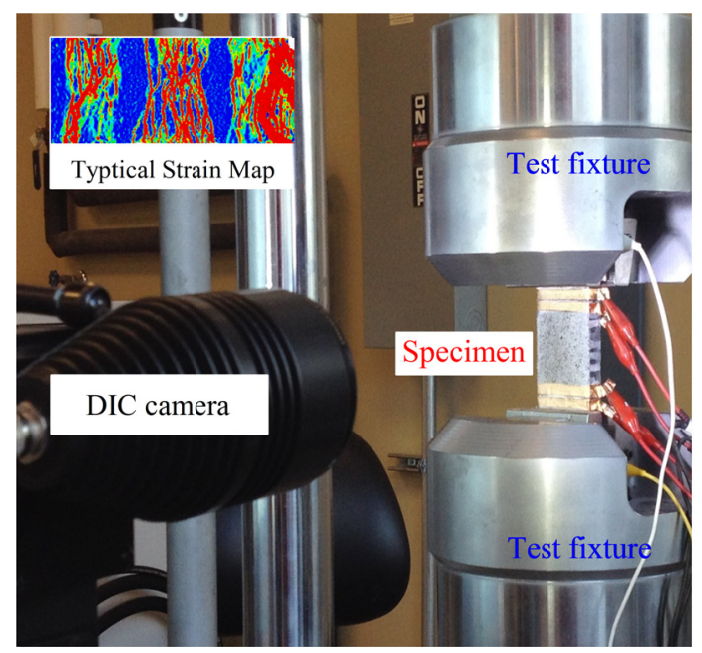

Figure 3: Measurement of crack opening and strain using a digital image correlation system (DIC). 


\subsection{Multiple microcracking}

The effect of multiple cracking process on the electrical response of HPFRCC was investigated through uniaxial tension test on unnotched coupon specimens. The test setup is shown in Figure 4. The region measured by DIC to generate tensile strain values during loading is shown in blue color. Four copper electrodes were attached to the surfaces of the specimen. The outer two electrodes had a spacing of $101.6 \mathrm{~mm}$, and were used to inject AC current. The inner two electrodes had a spacing of $66 \mathrm{~mm}$, and were used to measure voltage change during loading. The uniaxial tension test was conducted with displacement control at a rate of $0.0005 \mathrm{~mm} / \mathrm{s}$. There were two testing protocols: (1) The electrical response of the specimen during multiple cracking process was measured at a frequency of $1500 \mathrm{HZ}$ throughout the test. (2) Electrical impedance spectroscopy was also conducted on the specimen at increasing strain levels, with an $\mathrm{AC}$ frequency ranging from $0.1 \mathrm{HZ}$ to $10 \mathrm{MHZ}$.

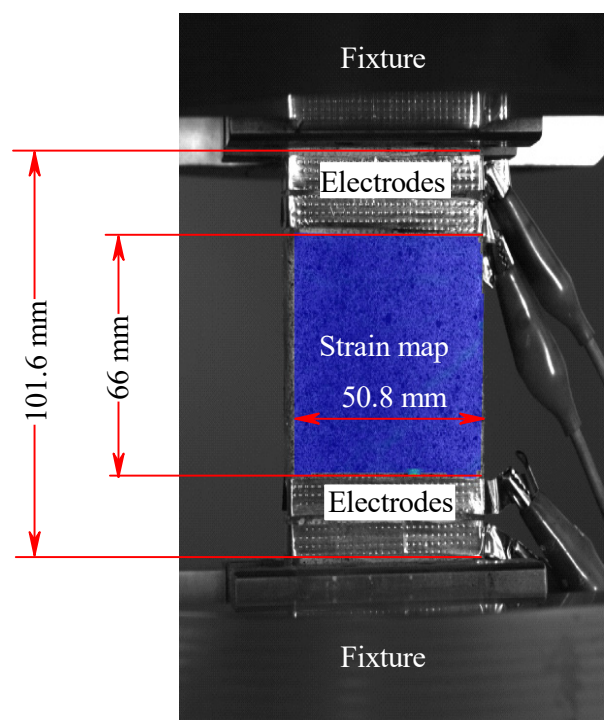

Figure 4: Uniaxial tension test setup and specimen geometry.

\section{RESULTS AND DISCUSSION}

\subsection{Effect of single crack opening on HPFRCC electrical response}

The fiber bridging tensile stress vs crack opening $(\sigma \sim \delta)$ relation, aka the fiber bridging "spring law", is shown in Figure 5. For both material designs, the values of complementary energy calculated from $\sigma \sim \delta$ curves were larger than crack tip energy of the matrices, thus leading to multiple cracking behavior and strain hardening in both materials. The peak fiber bridging capacity of HPFRCC 1 was higher than HPFRCC 2, with a lower critical crack opening corresponding to the peak fiber bridging stress. This was because HPFRCC 2 has a weaker interfacial bond between fibers and the cementitious matrix.

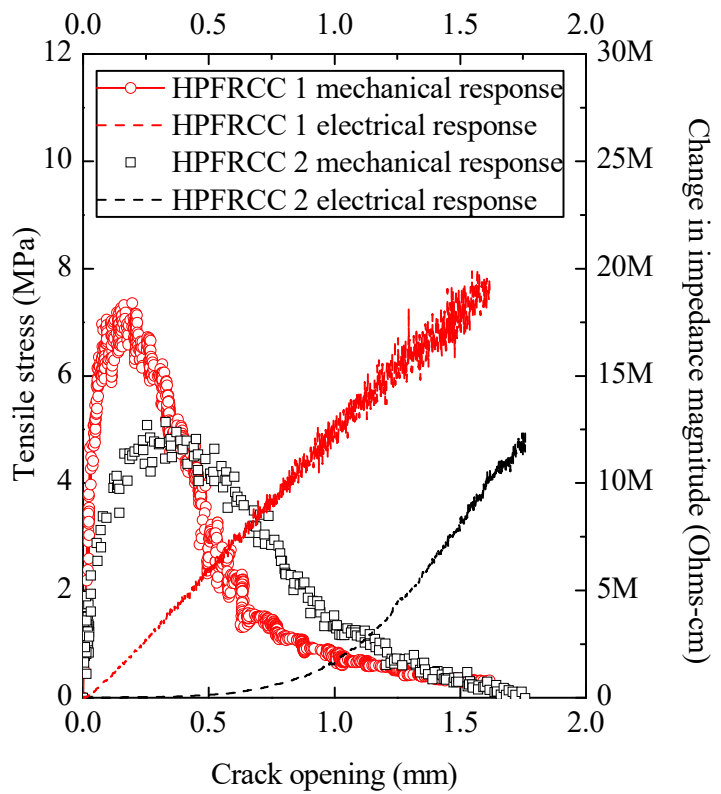

Figure 5: HPFRCC fiber bridging stress vs. crack opening relation ( $\sigma \sim \delta$ relation), and impedance magnitude change vs. crack opening relation.

For HPFRCC 1, a strong linear correlation was observed between impedance magnitude change and crack opening. However, for HPFRCC 2, a nonlinear relation was found. It is hypothesized that for HPFRCC 1, the crack can be represented by a parallel plate capacitor element. The distance between plates is related to crack opening. Because the fibers bridging the crack are nonconductive, the effect of a resistor element on the impedance magnitude is negligible. As a result, the impedance magnitude change is linearly related to the distance between plates in the capacitor element, and then linearly related to crack 
opening. For HPFRCC 2, it is hypothesized that the presence of conductive carbon black particles led to significant contact impedance at the fiber/matrix interfaces. Therefore, the increase of impedance magnitude during crack opening was due to the combined effects of change in resistance which depends on statistical fiber debonding, pullout and rupture behavior as the crack opens, and the change in capacitance which depends on the crack opening value; this combined effects led to a nonlinear relation between crack opening and impedance magnitude change.

\subsection{Effect of multiple microcracking on HPFRCC electrical response}

The uniaxial tensile stress-strain relations of the two HPFRCC materials are shown in Figure 6 and 7. Their impedance magnitude changes with increasing strain are also plotted. Both materials exhibited tensile strain hardening behavior, with a tensile strain capacity approximately 4\% for HPFRCC 1 and $5 \%$ for HPFRCC 2. As a brittle matrix composite material, the increasing strain is mainly a result of the increasing number of cracks without much increase in the width of each steady-state microcrack. For HPFRCC 1, the measured impedance magnitude change showed large noise; but a trend of increasing impedance magnitude with increasing strain was clear. For HPFRCC 2, the matrix electrical conductivity was improved through the incorporation of conductive nanoparticles, leading to little noise in the measured impedance. The impedance magnitude increased with increasing strain. This increase was slight during the elastic stage, became faster during the strain-hardening stage, and most predominant during the tension-softening stage. This phenomenon suggested that during elastic straining, the material exhibited a piezoresistive behavior. The strain of the material led to the change in the material's band structure, making it more difficult for electrons to be excited into the conduction band. The charge carrier density thus decreased, leading to the increase in material impedance magnitude. During the strain- hardening stage, multiple steady-stage cracking occurred. The increase of impedance magnitude during this stage was mainly a result of increasing crack number, without much change in crack width. According to the findings in Section 4.1, Figure 5, this impedance magnitude increase can be viewed as the increased number of resistors and capacitors with similar electrical parameters in the circuit system. During tension softening stage, the fast increase of impedance magnitude was dominated by localized Griffith crack opening, which can also be represented by impedance increase during the descending branch of the $\sigma \sim \delta$ relation.

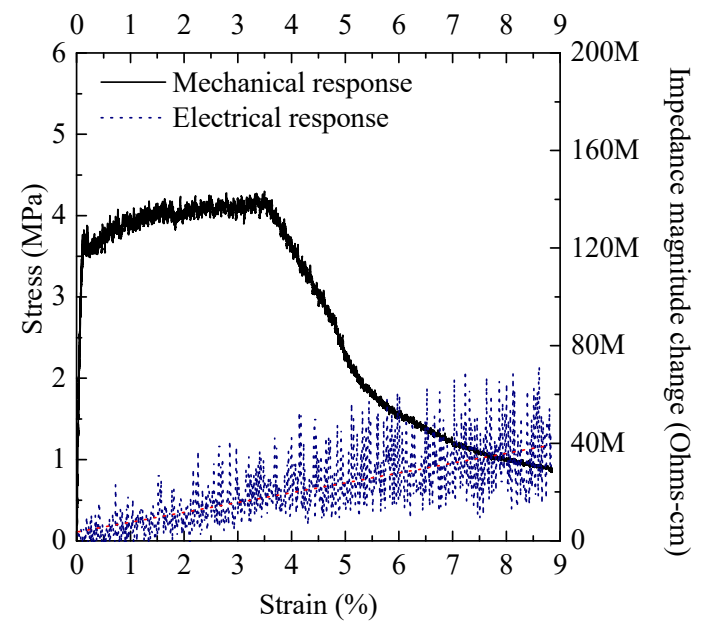

Figure 6: Uniaxial tension results on HPFRCC 1 and electrical response.

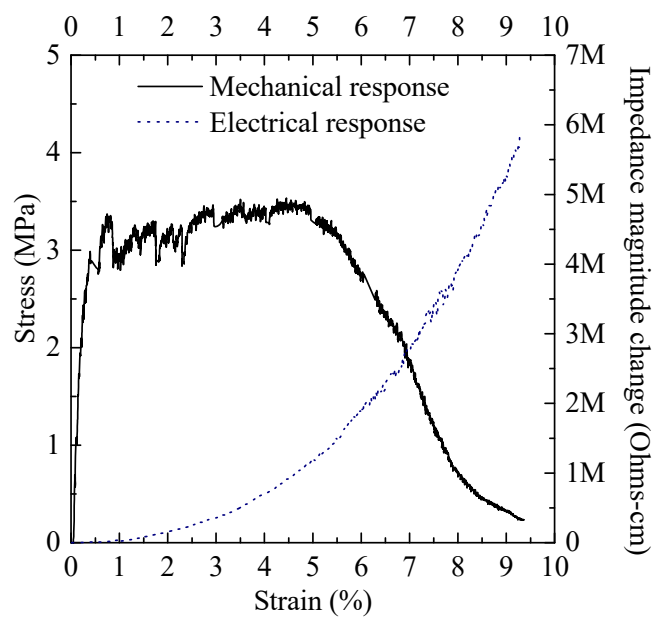

Figure 7: Uniaxial tension results on HPFRCC 2 and electrical response. 
The effect of multiple microcracking at increasing strain level on the complex impedance Nyquist plots of HPFRCC 2 in the complex plane is shown in Figure 8. The imperfect (distorted) semi-circular arc represents the electrical response of the material, while the "tail" segment represents the electrical response of material/electrode interface; the former is the point of interest of this study. It is shown that with increasing strain level and the number of steady-state microcracking, the radius of the arc became larger, and the origin of the arc at high frequencies shifted to the right. To explain this, equivalent circuit analysis was performed, which can provide estimates of the parameters of resistors and capacitors in the circuit system and lead to a quantitative analysis of HPFRCC electrical properties. The detailed analysis will be reported in a journal paper.

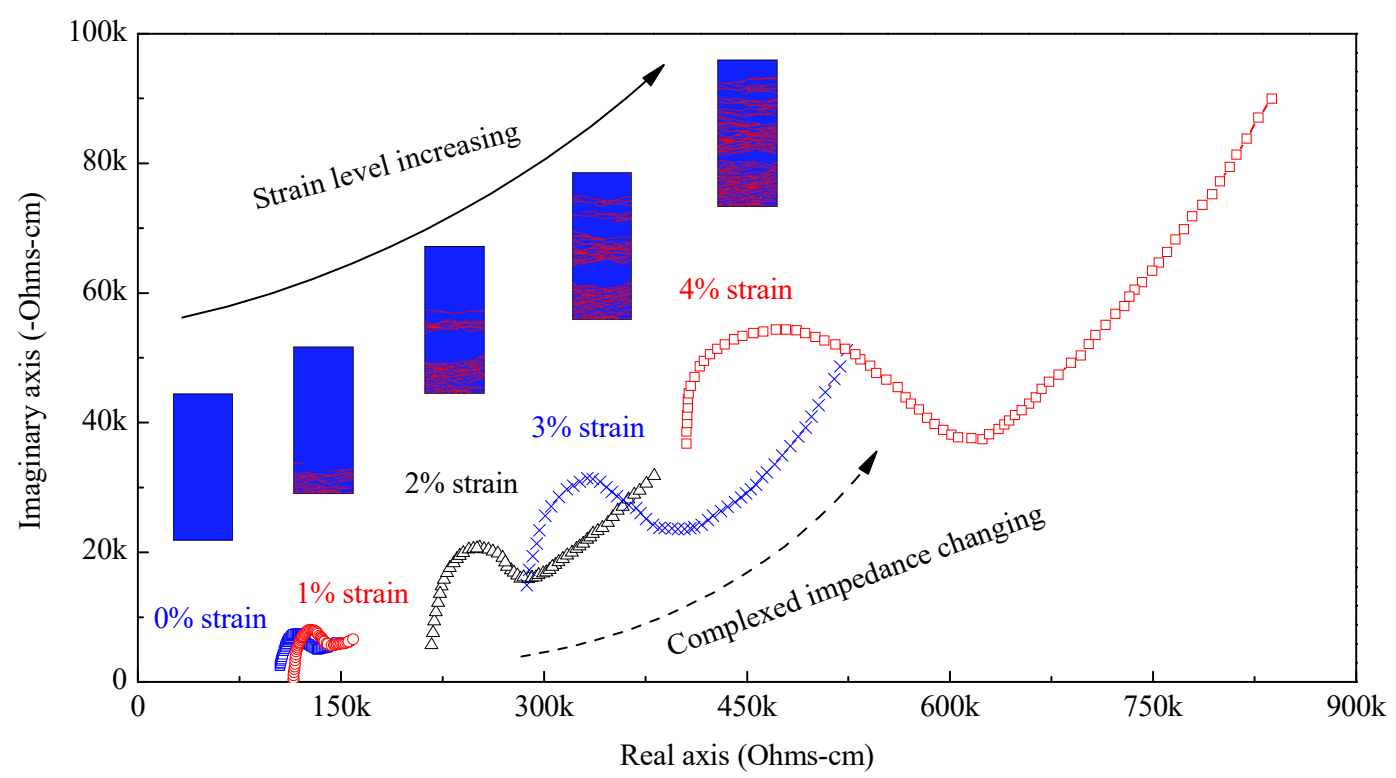

Figure 8: Effect of multiple microcracking on the Nyquist plot of HPFRCC 2.

\section{CONCLUSIONS}

Based on the experimental study on the effect of single crack opening and multiple cracking on HPFRCC electrical response, the following conclusions can be made:

1. During the opening of a single crack in HPFRCC, the crack opening value was strongly correlated to electrical impedance magnitude of the material. For HPFRCC 1 with nonconductive polymeric fibers, this relation was linear. For HPFRCC 2 with nonconductive polymeric fibers but a small amount of conductive nanoparticles dispersed in the cementitious matrix, the relation was nonlinear.

2. Crack in HPFRCC can be represented by a capacitor element. When the effect of resistor was negligible, which was the case for HPFRCC 1, the increase in impedance magnitude was linearly proportional to the crack opening which can be represented by the distance between the plates of the capacitor. When the effect of contact resistance was not negligible, which was the case for HPFRCC 2, the increase in impedance magnitude was due to a combined effect of increasing distance between crack surfaces as well as fiber debonding, pullout and rupture behavior with increasing crack width. The led to a nonlinear relation between the crack opening and impedance magnitude change.

3. HPFRCC 2 has improved electrical properties compared with HPFRCC 1, leading to significantly reduced noise in impedance measurements with increasingly applied strain. This indicated that HPFRCC 2 has a potential to act as a strain sensor. Its impedance magnitude increased with increasing strain; this increase was slower at the elastic stage, 
became faster during multiple steady-state cracking stage, and fastest during localized cracking stage. The sensitivity of the material electrical response during the three different stages made it a promising material to "selfsense" its strain and damage states.

4. HPFRCC 2 complex impedance Nyquist plot significantly changed with increasing number of multiple microcracks. This interesting phenomenon suggested further analysis and development of analytical models to represent the electromechanical behavior of HPFRCC 2.

\section{REFERENCES}

[1] Li, V.C., Wu, C., Wang, S., and Ogawa, A. 2013. Interface Tailoring for StrainHardening PVA-ECC. ACI Material Journal 99(5):464-472.

[2] Li, M., and Li, V.C. 2012. Rheology, fiber dispersion, and robust properties of engineered cementitious composites. Materials and Structures 46(3):405-420.

[3] Li, M., Lin, V., Lynch, J., and Li, V.C. 2013. Carbon Black Engineered Cementitious Composites - Mechanical and Electrical Characterization. ACI Special Publication 292:1-16.

[4] Li, M., Lin, V., Lynch, J., and Li, V. C. 2011. Multifunctional Carbon Black Engineered Cementitious Composites for the Protection of Critical Infrastructure. High-Performance Fiber Reinforced Cement Composites 6 2: 99-106.

[5] Xie, P., Gu, P., Xu, Z. and Beaudoin, J.J. 1993. A rationalized A.C. impedance Mmodel for microstructural characterization of hydrating cement systems. Cement and Concrete Research 23: 359-367.

[6] Cabezaa, M., Merinoa, P., Mirandab, A., No'voaa, X.R. and Sancheza, I. 2002. Impedance spectroscopy study of hardened Portland cement paste. Cement and Concrete Research 32: 881-891.

[7] Chu, T.C., Ranson, W.F., Sutton, M.A., and Peters, W.H. 2005. Applications of digital-image-correlation techniques to experimental mechanics. Experimental Mechanics 25 (3): 232-244. 